\title{
Corrigendum: Strategy and sustainability: a strategic logic for engagement with the environment
}

Mike Rosenberg

Correction to: Palgrave Communications (2015) 1, Article number: 15034. doi: 10.1057/palcomms.2015.34; Published 3 Nov 2015; Updated 15 Dec 2015

The following phrases on pages 3 and 4 featured an incorrect spelling.

On page 3, "Brake the Law" should have read "Break the Law".

On page 4, "Pay for Principal" should have read "Pay for Principle".

The article has been corrected online. 